\title{
Antibakterieller Wirkstoff schaltet gezielt Staphylococcus aureus aus
}

Das Biotechnologieunternehmen Micreos entwickelt zielgenaue Technologien gegen krankheitserregende Bakterien. Mit dem Enzym Staphefekt ${ }^{\mathrm{Tm}}$ ist ein Wirkstoff auf dem Markt, der nur Staphylococcus-aureus-Bakterien einschließlich seiner resistenten Variante MRSA abtötet und sich so gegenüber Antibiotika abgrenzt. In $\mathrm{Me}$ dizinprodukten der Marke Gladskin Eczema wird der Wirkstoff erfolgreich zur Behandlung entzündlicher Symptome der Neurodermitis eingesetzt.

Im Oktober 2015 hat Micreos unter Federführung des Deutschen Neurodermitis Bund e.V. (DNB) einen Produkttest mit 250 Mitgliedern durchgeführt, die über vier bis sechs Wochen Gladskin
Eczema Creme testen konnten. Die Auswertung von Fragebögen ergab, dass über $80 \%$ der Teilnehmer eine Linderung ihrer Symptome sahen. Vor allem Rötungen, trockene Haut und Juckreiz gingen zurück. Bei über $50 \%$ der Testpersonen, die zuvor regelmäßig Kortikosteroide (Kortison) zur Behandlung ihrer Neurodermitis einsetzten, ging der Bedarf unter Gladskin-Anwendung zurück. Ergebnisse des Tests werden in der aktuellen Ausgabe des Mitgliedermagazins des DNB (Hautfreund, Ausgabe 1/2016) zusammengefasst.

Staphefekt $\mathrm{t}^{\mathrm{TM}}$ ist ein Enzym (Endolysin), das gezielt artspezifische Verbindungen in der Bakterienhülle von $S$. aureus angreift und diese zum Platzen bringt. Anders als Antibiotika, die auch nützliche Bakterien der schützenden Hautflora treffen und selbst in niedrigen Dosierungen zu Resistenzen führen können, verschont das Enzym die übrige Hautflora. Es wirkt auch gegen multiresistente S.-aureus-Bakterien, ohne selbst zu Resistenzen zu führen.

Bei 70-90\% der Neurodermitiker lässt sich S. aureus in den Hautläsionen nachweisen. Inzwischen wird dieses Bakterium nicht mehr nur als verstärkender Faktor, sondern zunehmend auch als einer von mehreren ursächlichen Faktoren der Neurodermitis diskutiert. Die gezielte Wirkungsweise des Enzyms ohne negativen Einfluss auf die Hautflora und ohne Eingriff in das Immunsystem ermöglicht eine langfristige Erhaltungstherapie bei Neurodermitis. Nähere Informationen zur Technologie unter www. gladskin.com/de/content/technology.

Nach Informationen von Micreos Human Health

\section{Lichtschutz und Vitamin-D-Mangel}

Das Photodermatologie-Symposium von La Roche-Posay fand dieses Jahr in Kooperation mit der Hautklinik Mainz statt. Unter anderem wurde erörtert, ob ein konsequenter Lichtschutz einen Vitamin-D-Mangel hervorrufen kann. Zu niedrige Vitamin-D-Spiegel (< $50 \mathrm{nmol} / \mathrm{l})$ sind schädlich und zu vermeiden, erläuterte Prof. Thomas Schwarz, Kiel. Jedoch: „UV-Strahlung ist ungeeignet, Vitamin-D-Defizienzen zu beheben“, verdeutlichte Schwarz die Problematik, „denn UV-Strahlung ist das wichtigste Karzinogen für Hautkrebs“.

Eine australische Langzeitstudie [Green A. et al. J Clin Oncol 2011] belegte erstmals, dass regelmäßig angewendeter UVSchutz die Entwicklung von aktinischen Keratosen signifikant reduziert. Gleichzeitig zeigten die Ergebnisse, dass die VitaminD-Werte der Verumgruppe zwar erniedrigt, aber nicht unter Norm waren. Untersuchungen an Patienten mit erythropoetischer Proptoporphyrie bzw. Lupus erythematodes, die einen konsequenten UV-Schutz betreiben und sich nicht der Sonne aussetzen, zeigen, dass hier durchaus Defizienzen auftreten können. Dies könnte einigen Experten zufolge aber auch daran liegen, dass die Erkrankung per se einen Vitamin-D-Mangel verursacht. Eine Kontrolle der Vitamin-D-Werte - und bei Bedarf eine entsprechende orale Supplementation - ist bei solchen Patientengruppen empfehlenswert.

Auch eine künstliche UV-Bestrahlung wie in Solarien eignet sich nicht, einen Vitamin-D-Mangel zu beheben. Abgesehen von dem erhöhten Risiko der Hautkrebsentstehung können hier auch Nebenwirkungen auftreten wie Sonnenbrand oder polymorphe Lichtdermatose. Da die Eigensynthese der Haut von

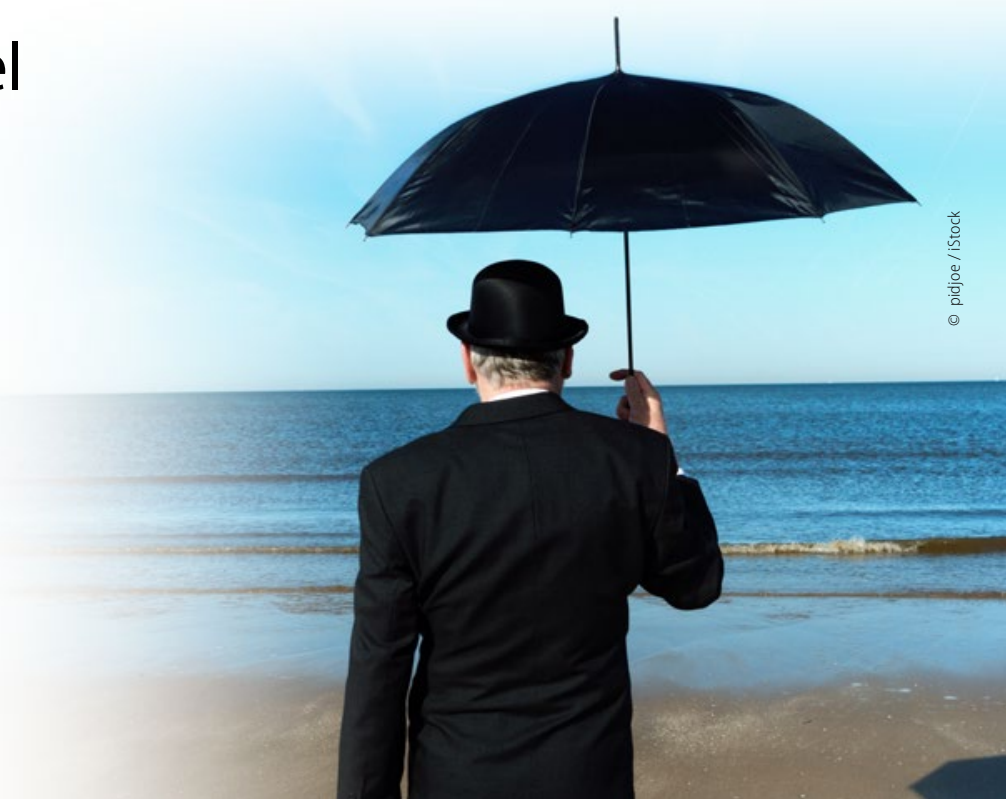

Personen, die einen konsequenten Lichtschutz betreiben, sollten auf ihren Vitamin-D-Spiegel achten.

Hauttyp, Alter, Bewölkung etc. abhängt, ist es zudem schwierig, konkrete Empfehlungen für natürliche Besonnung abzuleiten. „Natürliche und künstliche UV-Strahlung sind nicht geeignet, einen Vitamin-D-Mangel zu beheben, da sie nicht steuerbar sind. Eine orale Substitution ist gleich wirksam, aber risikoärmer und hat daher eindeutig Präferenz", fasste Schwarz zusammen.

Nach Informationen von La Roche-Posay 